\title{
ON INFINITE SERIES CONCERNING ZEROS OF BESSEL FUNCTIONS OF THE FIRST KIND
}

\author{
ANDREA GIUSTI ${ }^{1}$ AND FRANCESCO MAINARDI ${ }^{2}$
}

\begin{abstract}
A relevant result independently obtained by Rayleigh and Sneddon on an identity on series involving the zeros of Bessel functions of the first kind is derived by an alternative method based on Laplace transforms. Our method leads to a Bernstein function of time, expressed by Dirichlet series, that allows us to recover the RayleighSneddon sum. We also consider another method arriving at the same result based on a relevant formula by Calogero. Moreover, we also provide an electrical example in which this sum results to be extremely useful in order to recover the analytical expression for the response of the system to a certain external input.
\end{abstract}

\section{INTRODUCTION}

As discussed in our previous paper [5] dealing with a peculiar mathematical model in linear viscoelasticity, there are strong hints about the possibility of computing the sum of the infinite series

$$
S_{\nu}=\sum_{n=1}^{\infty} \frac{1}{j_{\nu, n}^{2}}=\frac{1}{4(\nu+1)}, \quad \nu>-1,
$$

where $j_{\nu, n}$ stands for the $n$th positive zero of the Bessel function of the first kind $J_{\nu}$. Indeed, in this note the proof is completely based on the Laplace transform method, generalizing our approach followed in [5].

In Section 2 we present a method that, being inspired by our paper [5], is based on the use of Laplace transform of a ratio of two modified Bessel functions $I_{\nu}$ of contiguous order $\nu>-1$. The inversion leads to positive increasing functions expressed by Dirichlet series that allows us to recover the Rayleigh-Sneddon result. These functions have a complete monotone derivative, so turn out to be Bernstein functions.

In Section 3 we discuss an application of the result within a class of models for electrical ladder networks dual to a class of viscoelastic models discussed in [4]. In particular, in this section we take advantage of a known analogy between viscoelastic and electrical systems which was widely discussed by Gross and Fuoss in $[6 ; 7 ; 8]$.

In Section 4 we briefly discuss an alternative method based on a relevant formula due to Calogero [3] of which we became aware only in the later stage of this work.

Concluding remarks are found in Section 5 where we outline the different motivation of the two approaches which appear to be somehow correlated. Moreover, in this section we also give some hints for potential future research concerning the analogous electrical model discussed in Section 3.

Detailed mathematical aspects of our approach discussed in Section 2 are presented in two Appendices for reader's convenience.

Date: June 21, 2016.

Key words and phrases. Dirichlet series - Laplace transform - Bessel functions.

Paper published in Eur. Phys. J. Plus (2016) Vol. 131:206, DOI: 10.1140/epjp/i2016-16206-4. 


\section{The Rayleigh-Sneddon Result Based on LAPlace transform}

Let us consider the following Laplace transform defined in the complex $s$-plane,

$$
\widetilde{F}_{\nu}(s)=\frac{2(\nu+1)}{s \sqrt{s}} \frac{I_{\nu+1}(\sqrt{s})}{I_{\nu}(\sqrt{s})}, \quad \nu>-1 .
$$

In view of the power series representation for the Modified Bessel functions of the first kind, see e.g. [1; 13]:

$$
I_{\nu}(z)=\left(\frac{z}{2}\right)^{\nu} \sum_{k=0}^{\infty} \frac{1}{k ! \Gamma(\nu+k+1)}\left(\frac{z}{2}\right)^{2 k}
$$

we recognize that $\widetilde{F}_{\nu}(s)$ turns out to be a single-valued function, with a simple pole in $s=0$ with residue 1 , and infinite poles in the points where

$$
I_{\nu}(\sqrt{s})=0 \quad \Longleftrightarrow \quad J_{\nu}(\lambda)=0,
$$

having renamed $\sqrt{s}=-i \lambda$ with $\lambda>0$ and denoted by $J_{\nu}$ the standard Bessel functions of the first kind. For $\nu>-1$ the zeros of $J_{\nu}$ are known to be all simple and real so that the poles of $\widetilde{F}_{\nu}(s)$ (different from zero) are located on the negative real axis. They are given by

$$
s_{\nu, n}=-j_{\nu, n}^{2}, \quad n=1,2, \ldots
$$

where $j_{\nu, n}$ stands for the $n$th positive zero of $J_{\nu}$. Of course, for $\widetilde{F}_{\nu}(s)$ the infinity is a singular point being a limit point of poles but for $\operatorname{Re}\{s\} \rightarrow+\infty$ the function goes to zero being

$$
\widetilde{F}_{\nu}(s) \sim 2(\nu+1) s^{-3 / 2}, \quad \operatorname{Re}\{s\} \rightarrow+\infty,
$$

as we recognize from the known asymptotic representation of the modified Bessel function

$$
I_{\nu}(z) \sim \frac{e^{z}}{\sqrt{2 \pi} z^{1 / 2}}, \quad|z| \rightarrow \infty,|\arg z|<\frac{\pi}{2} .
$$

Inverting $\widetilde{F}_{\nu}(s)$ by using the residue theorem, see for details Appendix A, we obtain the original function $F_{\nu}(t)$ (in the time domain) as

$$
F_{\nu}(t)=1-4(\nu+1) \sum_{n=1}^{\infty} \frac{\exp \left(-j_{\nu, n}^{2} t\right)}{j_{\nu, n}^{2}}, \quad t>0 .
$$

The series of exponentials in the R.H.S is a particular type of Dirichlet series of which we prove the absolute convergence for $t>0$ in Appendix B.

From this representation by means of a Dirichlet series we recognize that $F_{\nu}(t)$ is a locally integrable, positive and increasing function for $t>0$, with infinite a derivatives alternating in sign. The local integrability is easily proved by recognizing that

$$
F_{\nu}(t) \sim 4(\nu+1) \frac{t^{1 / 2}}{\sqrt{\pi}}, \quad t \rightarrow 0^{+},
$$

in virtue of the asymptotic behaviour of the Laplace transform $\widetilde{F}_{\nu}(s)$ outlined in Eq. (2.5).

Using a precise mathematical terminology, the function $F_{\nu}(t)$ is classified as a Bernstein function and its derivative $F_{\nu}^{\prime}(t)$ as a completely monotone $(\mathrm{CM})$ function. For more details on these classes of functions we may refer the interested reader to the excellent monograph by Schilling et al. [11].

Now setting to zero the limiting value $F_{\nu}\left(0^{+}\right)$in Eq. (2.7) we promptly derive the result by Rayleigh and Sneddon expressed in Eq. (1.1). 


\section{An ELECTRICAL APPLiCATION BASED ON ELECTRO-MECHANiCAL ANALOGIES}

In 1956, B. Gross and R. M. Fuoss first presented a formal correspondence between the analytical description of electrical ladder structures and viscoelastic systems. In particular, in [6] they considered a model for the deformation of an elastic rod in a viscous medium. To be more specific, let $\sigma=\sigma(t, x)$ and $\varepsilon=\varepsilon(t, x)$ be the stress and the strain functions at a point $x$ and a time $t$, respectively. Then, the viscoelastic model in [6] is given by the following stress-strain relations:

$$
\sigma=e \frac{\partial \varepsilon}{\partial x}, \quad \frac{\partial \sigma}{\partial x}=\eta \frac{\partial \varepsilon}{\partial t}
$$

where $1 / e$ and $\eta$ are respectively the spring modulus and the viscosity constant.

Clearly, a priori such a model would not be linear, but it can be linearized (as above) by considering the case of small deformations. As argued in [6], truly linear systems can be given in terms electrical analogs of mechanical models. Indeed, an example of electrical analog to the viscoelastic system discussed by Gross and Fuoss is provided by a finite cable in which a uniform wire of specific resistance $\rho$ is enclosed into a dielectric material of fixed thickness. For such an electrical system we have that:

$$
I=\frac{1}{\rho} \frac{\partial V}{\partial x}, \quad \frac{\partial I}{\partial x}=c \frac{\partial V}{\partial t}
$$

where $I=I(t, x)$ and $V=V(t, x)$ are respectively the current and the potential. Moreover, $\rho$ and $c$ are respectively the resistance and the capacitance per unit of length.

Now, comparing Eq. (3.1) and Eq. (3.2) we can immediately read off the prescriptions commonly used to implement electro-mechanical analogies. Indeed, as discussed in $[6 ; 7 ; 8]$, we heve

$$
\left.\begin{array}{l}
\sigma \text { stress } \\
\varepsilon \text { strain } \\
E \text { elastic modulus } \\
\eta \text { viscosity }
\end{array}\right\} \Longleftrightarrow\left\{\begin{array}{l}
I \text { current } \\
V \text { potential } \\
1 / R \text { conductance } \\
C \text { capacitance }
\end{array}\right.
$$

Now, if we start off with a more general viscoelastic system, its corresponding analogous electrical system would not be described simply in terms of single electrical component. Indeed, in general we have that a viscoelastic system formally corresponds to a class of electrical ladder networks given by the following formal duality

$$
\left.\begin{array}{l}
\text { Spring } \\
\text { Dashpot }
\end{array}\right\} \Longleftrightarrow\left\{\begin{array}{l}
\text { Resistor } \\
\text { Capacitor }
\end{array}\right.
$$

Let us consider the function $\widetilde{F}_{\nu}(s)$ in Eq. (2.1). As discussed in [4], the function

$$
\widetilde{\Phi}_{\nu}(s)=s \widetilde{F}_{\nu}(s)=\frac{2(\nu+1)}{\sqrt{s}} \frac{I_{\nu+1}(\sqrt{s})}{I_{\nu}(\sqrt{s})}, \quad \nu>-1
$$

represents the Laplace transform of the relaxation memory function $\Phi_{\nu}(t)$ for a general class of viscoelastic models. Then in time domain we get by using Eq.(2.7)

$$
\Phi_{\nu}(t)=\frac{d F_{\nu}}{d t}(t)=4(\nu+1) \sum_{n=1}^{\infty} \exp \left(-j_{\nu, n}^{2} t\right) .
$$

Following the general description of linear viscoelasticity displayed in [10] for bodies in rest for $t<0$, one immediately gets the stress-strain relation in the Laplace domain for 
the class of models corresponding to the relaxation memory function in Eq. (3.3), i.e.

$$
\widetilde{\sigma}(s)=s \widetilde{G}_{\nu}(s) \widetilde{\varepsilon}(s)=\left(1-\widetilde{\Phi}_{\nu}(s)\right) \widetilde{\varepsilon}(s)
$$

where $\widetilde{G}_{\nu}(s)$ is the Laplace transform of the so called relaxation modulus $G_{\nu}(t)$ with a finite limiting value at $t=0$. We note that, for the sake of convenience, we consider all quantities in non-dimensional form after a suitable scaling so that we agree to take $G_{\nu}\left(0^{+}\right)=1$. Inverting $\widetilde{G}_{\nu}(s)$ we get

$$
G_{\nu}(t)=1-\int_{0}^{t} \Phi_{\nu}(\tau) d \tau=1-4(\nu+1) \sum_{n=1}^{\infty} \frac{1-\exp \left(-j_{\nu, n}^{2} t\right)}{j_{\nu, n}^{2}} .
$$

Using Eq. (1.1) this expression immediately turns into

$$
G_{\nu}(t)=4(\nu+1) \sum_{n=1}^{\infty} \frac{\exp \left(-j_{\nu, n}^{2} t\right)}{j_{\nu, n}^{2}} .
$$

Alternatively we can get $G_{\nu}(t)$ from Eq. (2.7) noting

$$
G_{\nu}(t)=1-F_{\nu}(t) .
$$
by

Now, the stress-strain relation in the time domain and in non-dimensional form is given

$$
\sigma(t)=\varepsilon(t)+\left(\dot{G}_{\nu} * \varepsilon\right)(t),
$$

where $*$ represents the convolution product ${ }^{1}$ and the dot represents the derivative with respect to the argument of the function. If we then consider the electro-mechanical analogy discussed above we get

$$
I(t)=V(t)+\left(\dot{G}_{\nu} * V\right)(t),
$$

which represents a characteristic equation for some peculiar electrical ladder networks dual to the viscoelastic model introduced above. From the mathematical and physical points of view, it is interesting to study the response of the current $I(t)$ to a step potential, i.e. $V(t)=\Theta(t)$. Therefore, plugging the input potential into Eq. (3.10) and integrating by parts we get

$$
I(t)=G_{\nu}(t)=4(\nu+1) \sum_{n=1}^{\infty} \frac{\exp \left(-j_{\nu, n}^{2} t\right)}{j_{\nu, n}^{2}},
$$

which clearly describes a relaxation process featured by a completely monotone behavior.

The response of the current is thus decaying exponentially for large times. However it is surprising to note that the initial decay rate of the current expressed by its time derivative

$$
\frac{d I}{d t}=\frac{d G_{\nu}}{d t}=-\Phi_{\nu}(t)
$$

is very strong because from Eq. (2.8) we get

$$
\Phi_{\nu}(t) \sim 2(\nu+1) \frac{t^{-1 / 2}}{\sqrt{\pi}}, \quad t \rightarrow 0^{+} .
$$

\footnotetext{
${ }^{1}$ Let $f(t), g(t)$ be causal functions (i.e. $f(t) \equiv f(t) \Theta(t)$, where $\Theta(t)$ is the Heaviside distribution), as in case of our concern, we have that

$$
(f * g)(t)=\int_{\mathbb{R}} f(t-\tau) g(\tau) d \tau=\int_{0}^{t} f(t-\tau) g(\tau) d \tau
$$
}


This asymptotic behaviour at $t=0^{+}$is peculiar of our electro-mechanical system; it is essentially due to the infinite (but discrete) distribution of simple relaxation elements of the Dirichlet series. In other words our Dirichlet series generalizes the finite Prony series commonly adopted in the analysis of relaxing electrical and mechanical systems.

\section{The Rayleigh-Sneddon sum Based on Calogero's formula}

We note that Eq.(1.1) is a particular case of more general formulas due to Lord Rayleigh in 1874, reported in the classical treatise by Watson [14], page 502, concerning the zeros of the Bessel functions. Related formulas were proved in 1960 by Sneddon [12] by a different approach, including again our Eq. (1.1).

Recently we became aware of the paper by Baricz et al [2] in which the authors have pointed out a formula derived in 1977 by Calogero [3] from which we can derive our particular case of the general results by Rayleigh and Sneddon after a few passages. Indeed, Calogero, based on the well known infinite product representation of the Bessel functions of the first kind

$$
J_{\nu}(x)=\frac{(x / 2)^{\nu}}{\Gamma(\nu+1)} \prod_{n=1}^{\infty}\left(1-\frac{x^{2}}{j_{\nu, n}^{2}}\right), \quad \nu>-1,
$$

and by means of an equivalent form of the Mittag-Leffler expansion, proved that

$$
\frac{J_{\nu+1}(x)}{J_{\nu}(x)}=\sum_{n=1}^{\infty} \frac{2 x}{j_{\nu, n}^{2}-x^{2}}, \quad \nu>-1 .
$$

Then, let us take the following limit as $x \rightarrow 0$

$$
\lim _{x \rightarrow 0} \frac{1}{2 x} \frac{J_{\nu+1}(x)}{J_{\nu}(x)}=\sum_{n=1}^{\infty} \frac{1}{j_{\nu, n}^{2}}=S_{\nu}, \quad \nu>-1 .
$$

Now, recalling the Taylor expansion of the Bessel functions of the first kind,

$$
J_{\nu}(z)=\left(\frac{z}{2}\right)^{\nu} \sum_{k=0}^{\infty} \frac{(-1)^{k}}{k ! \Gamma(\nu+k+1)}\left(\frac{z}{2}\right)^{2 k},
$$

the limit in Eq. (4.3) turns out to be after simple calculations

$$
\lim _{x \rightarrow 0} \frac{1}{2 x} \frac{J_{\nu+1}(x)}{J_{\nu}(x)}=\frac{1}{4(\nu+1)}, \quad \nu>-1 .
$$

Comparing the two limits in Eqs. (4.3), (4.5) we obtain the Rayleigh-Sneddon sum.

\section{Concluding REMarks}

It is straightforward to note that the derivation of the Rayleigh-Sneddon result discussed in Section 4 is more direct than ours based on the Laplace transforms, presented in Section 2. By the way our method allows to define locally integrable functions of time, $F_{\nu}(t)$, expressed as Dirichlet series related to the positive zeros of the Bessel functions of the first kind of order $\nu>-1$. We recognize that these functions are of Bernstein type, and thus suitable to characterize creep processes in linear viscoelasticity, see e.g. [10]. As a consequence, their derivatives (with minus sign) are thus completely monotonic and hence suitable for relaxation processes in electro-mechanical systems as outlined along this paper.

The two methods, even though they appear to correlated, arise from different motivations. 
On the one hand, the approach discussed in Section 4 is essentially based on a noteworthy formula by Calogero that was motivated by the researches about the connection between the motion of poles and zeros of special solutions of partial differential equations and many-body problems as outlined in [2].

On the other hand, our approach discussed in Section 2 was inspired by our analysis dealing with a peculiar mathematical model in linear viscoelasticity, see [5]. This model was characterized in the Laplace domain by ratios of modified Bessel of contiguous order 0,1 and 1,2, leading to completely monotone and Bernstein functions. In [5] these functions were expressed in terms of Dirichlet series involving the positive zeros of the Bessel functions $J_{0}$ and $J_{2}$. Extending to generic orders $\nu>-1$ but always using the Laplace transforms, in the present note we are able to prove the mathematical result by Rayleigh and Sneddon but also, in a forthcoming work [4], to introduce a new class of viscoelastic models.

Finally, in Section 3 we have been able to provide a general class of electrical ladder networks featuring the properties of being dual to a general class of viscoelastic media described in [4]. Then, it would be interesting for future research to investigate the dynamical evolution equations in space and time arising from these kinds of electro-mechanical models.

Acknowledgements. The authors would like to thank Ivano Colombaro for the many discussions on the preliminary draft of this paper. We are also grateful to Arpad Baricz for pointing out some interesting historical facts connected with Eq. (1.1) and to the anonymous reviewer for the thorough reading and constructive comments on our manuscript. The work of A.G. and F. M. has been carried out in the framework of the activities of the National Group for Mathematical Physics (GNFM, INdAM).

\section{Appendix A}

Here we show the details how to obtain $F_{\nu}(t)$ in Eq. (2.7) by inverting the Laplace transform $\widetilde{F}_{\nu}(s)$ in Eq. (2.1). We need to evaluate the Bromwich Integral:

$$
F_{\nu}(t)=\frac{1}{2 \pi i} \int_{B r} \widetilde{F}_{\nu}(s) e^{s t} d s
$$

by applying the Residue Theorem.

Proof. Let us first note that $s=0$ is a simple pole for $\widetilde{F}_{\nu}(s)$ whose residue is 1 as it is deduced by computing the limit of $s \widetilde{F}_{\nu}(s)$ as $s \rightarrow 0$. In fact this result is easily obtained by taking the first term of the Taylor series (2.2) for $I_{\nu}$ and $I_{\nu+1}$ around $s=0$.

Let us then recall that the simple poles $s_{\nu, n}$ exhibited by $\widetilde{F}_{\nu}(s)$ on the negative real axis are the zeros of $I_{\nu}(\sqrt{s})$ and hence related to the positive zeros $j_{\nu, n}$ of the Bessel function of the first kind $J_{\nu}(z)$ via Eq. (2.4), that we repeat hereafter for convenience:

$$
s_{\nu, n}=-j_{\nu, n}^{2}, \quad n=1,2, \ldots
$$

We thus have

(A3) $F_{\nu}(t)=1+\sum_{s_{\nu, n}} \mathcal{R} e s\left\{\widetilde{F}_{\nu}(s) e^{s t}\right\}_{s=s_{\nu, n}}=1+\sum_{n=1}^{\infty} \mathcal{R} e s\left\{\frac{2(\nu+1)}{s \sqrt{s}} \frac{I_{\nu+1}(\sqrt{s})}{I_{\nu}(\sqrt{s})} e^{s t}\right\}_{s=s_{\nu, n}}$, 
where the $n$-th residue can be computed as follows:

$$
\begin{aligned}
& \mathcal{R} e s\left\{\frac{2(\nu+1)}{s \sqrt{s}} \frac{I_{\nu+1}(\sqrt{s})}{I_{\nu}(\sqrt{s})} e^{s t}\right\}_{s=s_{\nu, n}}=\lim _{s \rightarrow s_{\nu, n}}\left(s-s_{\nu, n}\right) \frac{2(\nu+1)}{s \sqrt{s}} \frac{I_{\nu+1}(\sqrt{s})}{I_{\nu}(\sqrt{s})} e^{s t} \\
& =\lim _{s \rightarrow s_{\nu, n}} \frac{2(\nu+1)}{s \sqrt{s}} \frac{I_{\nu+1}(\sqrt{s})}{I_{\nu}^{\prime}(\sqrt{s}) /(2 \sqrt{s})} e^{s t}=4(\nu+1) \frac{\exp \left(s_{\nu, n} t\right)}{s_{\nu, n}} .
\end{aligned}
$$

Above we have used the property that if $s_{\nu, n}$ is a zero of the modified Bessel function $I_{\nu}$, then we have,

$$
I_{\nu}^{\prime}\left(s_{\nu, n}\right)=I_{\nu+1}\left(s_{\nu, n}\right),
$$

as we deduce from the general identity for modified Bessel functions, see e.g. [1],

$$
I_{\nu}^{\prime}(z)=\frac{\nu}{z} I_{\nu}(z)+I_{\nu+1}(z) .
$$

Thus, we finally get

$$
F_{\nu}(t)=1+\sum_{n=1}^{\infty} \mathcal{R} e s\left\{\widetilde{F}_{\nu}(s) e^{s t} ; s=-j_{\nu, n}^{2}\right\}=1-4(\nu+1) \sum_{n=1}^{\infty} \frac{\exp \left(-j_{\nu, n}^{2} t\right)}{j_{\nu, n}^{2}} .
$$

in agreement with Eq. (2.7).

\section{Appendix B}

Here we show that the Dirichlet series (2.7) is absolutely convergent for $t>0$ basing the proof on a statement of the classical treatise by Hardy and Riesz [9].

Proof. Consider a Dirichlet series in the $z$-complex plane:

$$
f(z)=\sum_{n=1}^{\infty} a_{n} \exp \left(-\alpha_{n} z\right), \quad z \in \mathbb{C} .
$$

We have convergence and absolute convergence in the right half planes $\operatorname{Re}\{z\}>\sigma_{c}$ and $\operatorname{Re}\{z\}>\sigma_{a}$, respectively, with $\sigma_{a} \geqslant \sigma_{c}$. The abscissa of convergence $\sigma_{c}$ and the abscissa of absolute convergence $\sigma_{a}$ satisfy the following condition:

$$
0 \leqslant \sigma_{a}-\sigma_{c} \leqslant d=\limsup _{n \rightarrow \infty} \frac{\ln n}{\alpha_{n}} .
$$

If $d=0$, then

$$
\sigma \equiv \sigma_{c}=\sigma_{a}=\limsup _{n \rightarrow \infty} \frac{\ln \left|a_{n}\right|}{\alpha_{n}} .
$$

In the case of our concern, $a_{n}=1 / j_{\nu, n}^{2}$ and $\alpha_{n}=j_{\nu, n}^{2}$. Then, we have to understand the behaviour of the coefficients $j_{\nu, n}$ for $n \gg 1$.

Considering the asymptotic representation:

$$
J_{\nu}(x) \stackrel{x \gg 1}{\sim} \sqrt{\frac{2}{\pi x}} \cos \left(x-\frac{(2 \nu+1) \pi}{4}\right)+o\left(x^{-3 / 2}\right),
$$

we can eventually get to the following conclusion:

$$
J_{\nu}\left(j_{\nu, n}\right)=0, \text { for } n \gg 1 \quad \Longrightarrow \quad j_{\nu, n} \propto n, \text { for } n \gg 1 \text {. }
$$

Thus,

$$
\frac{\ln n}{\alpha_{n}}=\frac{\ln n}{j_{\nu, n}^{2}} \stackrel{n \gg 1}{\sim} \frac{\ln n}{n^{2}} \stackrel{n \rightarrow \infty}{\longrightarrow} 0
$$


from which we deduce that $d=0$.

Finally,

$$
\sigma \equiv \sigma_{c}=\sigma_{a}=\limsup _{n \rightarrow \infty} \frac{\ln \left|a_{n}\right|}{\alpha_{n}}=0 .
$$

This result allows us to conclude that the Dirichlet series in Eq. (2.7) is absolutely convergent for $t>0$.

\section{REFERENCES}

[1] M. Abramowitz and I.A. Stegun, Handbook of Mathematical Functions, Dover, New York (1965).

[2] Á. Baricz, D.J. Maširević, T.K. Pogány, R.Szász, On an identity for zeros of Bessel functions, J. Math. Anal. Appl. 422 (2015), 27-36. DOI: 10.1016/j.jmaa.2014.08.014

[3] F. Calogero, On the zeros of Bessel functions, Lett. Nuovo Cimento 20 No 7 (1977), 254-256.

[4] I. Colombaro, A. Giusti, F. Mainardi, A class of linear viscoelastic models based on Bessel functions, Meccanica, published online, 23 May 2016. DOI: 10.1007/s11012-0160456-5. [E-print arXiv:1602.04664]

[5] A. Giusti, F. Mainardi, A dynamic viscoelastic analogy for fluid-filled elastic tubes, Meccanica, published online, 04 February 2016. DOI: 10.1007/s11012-016-0376-4. [Eprint arXiv:1505.06694]

[6] B. Gross, R.M. Fuoss, Ladder structures for representation of viscoelastic systems, J. Polymer Science 19 (1956), 39-50.

[7] B. Gross, Ladder structures for representation of viscoelastic systems, II, J. Polymer Science 20 (1956), 121-131.

[8] B. Gross , Electrical analogs for viscoelastic systems, J. Polymer Science 20 (1956), $371-380$.

[9] G.H. Hardy, M. Riesz, The General Theory of Dirichlet Series, Cambridge University Press, Cambridge (1915).

[10] F. Mainardi, Fractional Calculus and Waves in Linear Viscoelasticity, Imperial College Press \& World Scientific, London and Singapore (2010).

[11] R.L. Schilling, R. Song, Z. Vondracek, Bernstein Functions. Theory and Applications, 2-nd Edition, De Gruyter, Berlin (2012).

[12] I. N. Sneddon, On some infinite series involving the zeros of Bessel functions of the first kind, Proc. Glasgow Math. Assoc. 4 (1960), 144-156.

[13] N. M. Temme, Special Functions: An Introduction to the Classical Functions of Mathematical Physics, Wiley-Interscience, New York (1996).

[14] G.N. Watson A treatise on the Theory of Bessel Functions, Cambridge University Press, Cambridge (1944).

\footnotetext{
${ }^{1}$ Department of Physics \& Astronomy, University of Bologna and infN. Via Irnerio 46, BOLOGNA, ITALY.

E-mail address: andrea.giusti@bo.infn.it

${ }^{2}$ Department of Physics \& Astronomy, University of Bologna and infN. Via Irnerio 46, BOLOGNA, ITALY.

E-mail address: francesco.mainardi@bo.infn.it
} 